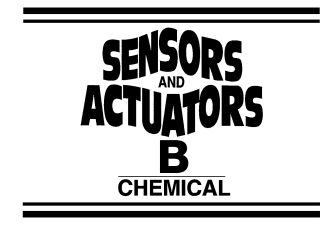

\title{
A microsensor array for biochemical sensing
}

\author{
Filip Van Steenkiste a,*, Kris Baert ${ }^{\text {a }}$, Dirk Debruyker ${ }^{\mathrm{b}}$, Vincent Spiering ${ }^{\mathrm{c}}$, \\ Bart van der Schoot ${ }^{d}$, Philippe Arquint ${ }^{d}$, Reinhard Born ${ }^{e}$, Klaus Schumann ${ }^{f}$ \\ a IMEC, MAP MST Division, Kapeldreef 75, B-3001 Leuven, Belgium \\ ${ }^{\mathrm{b}}$ ESAT, Katholieke Universiteit Leuven, Lueven, Belgium \\ ${ }^{\mathrm{c}}$ MESA Research Institute, University of Twente, Twente, Netherlands \\ ${ }^{\mathrm{d}}$ IMT, Institute of Microtechnology, University of Neuchâtel, Neuchâtel, Switzerland \\ e Dornier, Daimler-Benz Aerospace, Friedrichshafen, Germany \\ ${ }^{\mathrm{f}}$ Hans-Knöll-Institut, Jena, Germany
}

Received 17 July 1997; accepted 18 July 1997

\begin{abstract}
A microsensor array to measure chemical properties of biological liquids is presented. A hybrid integration technique is used to mount four sensor chips on a micro flow channel: a pressure, temperature, $\mathrm{pH}$, combined $\mathrm{pO}_{2}$ and $\mathrm{pCO}_{2}$ sensor chip. This results in a microsensor array which is developed to meet the technical requirements for space applications. The integration method allows to integrate other types of sensor chips. This multi-purpose and multi-user approach makes the microsensor array suitable for various biochemical applications. (C) 1997 Published by Elsevier Science S.A.
\end{abstract}

Keywords: Microsensor array; Hybrid; Integration method; Biological liquids

\section{Introduction}

Over the past years there has been an increasing interest in flow type biosensing devices, which take up samples at a constant rate and perform an in situ or in vivo monitoring. Recent progress made it possible to fabricate three-dimensional microstructures, e.g. by using micromachining. This allows the development of miniaturized flow type biosensing devices, which take up minimum amounts of biological samples. The microsensor array, presented in this paper, is a flow type sensor array for space applications.

Since the microsensor array operates in a biological environment, biocompatibility is one of the main requirements for this instrument. This means that the microsystem does not influence the characteristics of the liquid sample being investigated. Another requirement is that low temperature packaging and mounting processes must be used in order not to damage the sensor chips. Also the possibility to visually inspect the liquid in the microsystem is required. Further, for space

\footnotetext{
* Corresponding author. Tel.: + 3216281 265; fax: + 3216281 501; e-mail: vnsteenk@imec.be
}

application one should keep the weight and volume of the system very low. For the same reason, the sample volume has to be kept small in order to limit reagent consumption. In the case of the microsensor array, the microsystem volume has been reduced to less than 17 $\mu 1$.

The microsensor array consists of the following modules:

- Flow system board: a board which contains the complete liquid handling system including a sensor carrier

- Sensor carrier: a micromachined structure which contains four sensor chips

- Interface electronics and data acquisition module

\section{Flow system board}

An overview of the flow system board layout is given in Fig. 1. This board contains the complete flow system: two magnetic valves, a peristaltic pump and a sensor carrier, which are interconnected by short tubes.

By switching the valves, it is possible to pump up three different liquids in sequence to the sensor carrier. 


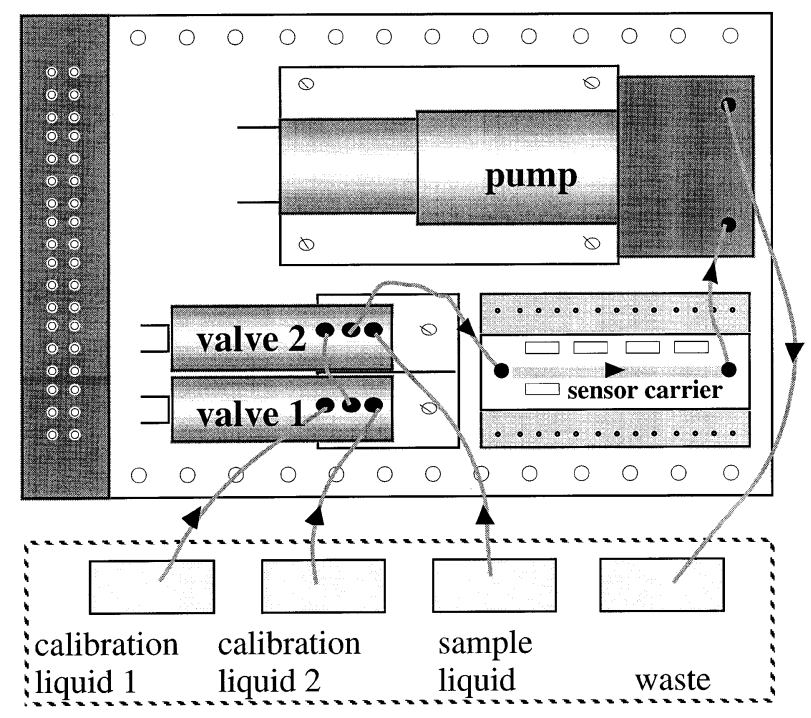

Fig. 1. Flow system board layout $(2 \times 3$ in $)$.

Before measuring the chemical properties of a sample, calibration liquids from the reservoirs are injected in the sensor carrier via the valves. From the outlet of the sensor carrier, the liquid is pumped into the waste reservoir. After calibration, the valves are switched and the sample liquid is pumped into the sensor carrier at a constant rate. While the sample in the sensor carrier is continuously renewed, the superfluous liquid is pumped into the waste.

The sensor carrier with the sensor chips is the core element of the flow system. The sensor carrier is mounted on a small PCB support which is plugged with pins on the flow system board. The sensor signals are read out via a flat cable connector. A demonstrator flow system board is shown in Fig. 2.

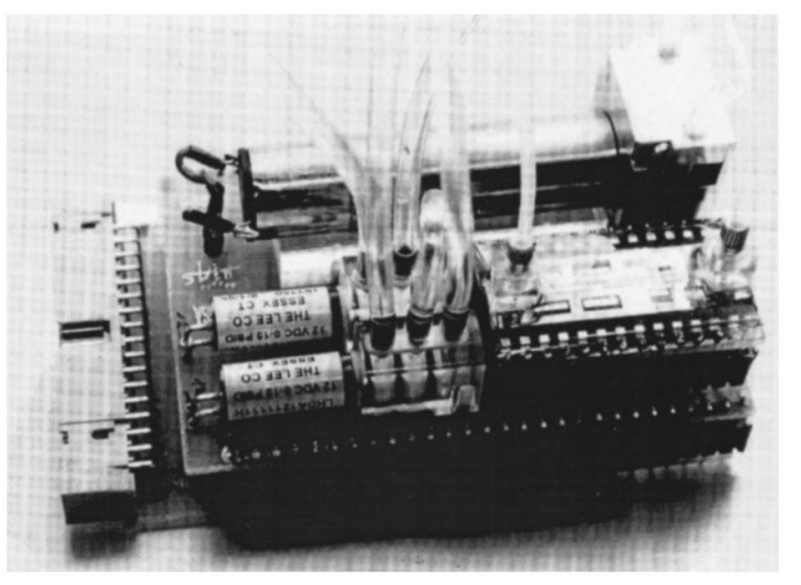

Fig. 2. Flow system board.

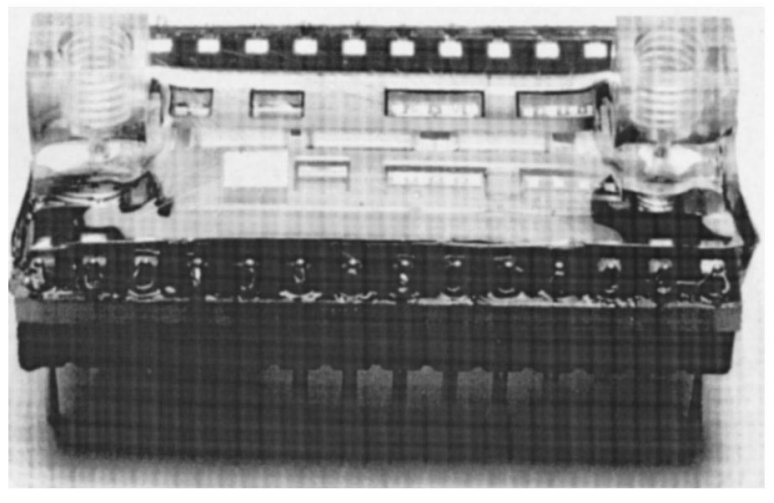

Fig. 3. Sensor carrier mounted on a PCB support with SIL connectors.

\section{Sensor carrier}

Fig. 3 shows a demonstrator sensor carrier which is mounted on a PCB support with SIL connectors. Two plexiglass modules, which have screwthread connection for the tubes, are sealed on the liquid inlet and outlet of the sensor carrier.

The sensor carrier (Fig. 4) consists of an internal liquid channel with a liquid inlet and outlet, which provides a continuous flow of liquid. The internal liquid channel (depth: $350 \mu \mathrm{m}$, width: $1400 \mu \mathrm{m}$ ) is formed by anodic bonding of a micromachined glass wafer to a micromachined silicon wafer. The individual sensor carriers are obtained by dicing this stack. Four sensor chips are mounted along the liquid channel on the back side of the sensor carrier.

Fig. 5 gives a cross section of the sensor carrier along the liquid channel and shows how the liquid is lead to the sensors by the shape of the pyrex glass.

Fig. 6 shows a cross section of the sensor carrier perpendicular to the liquid channel. The sensors are in contact with the internal liquid channel by through holes in the silicon. The four sensor chips are sealed hermetically to the silicon side by means of epoxy

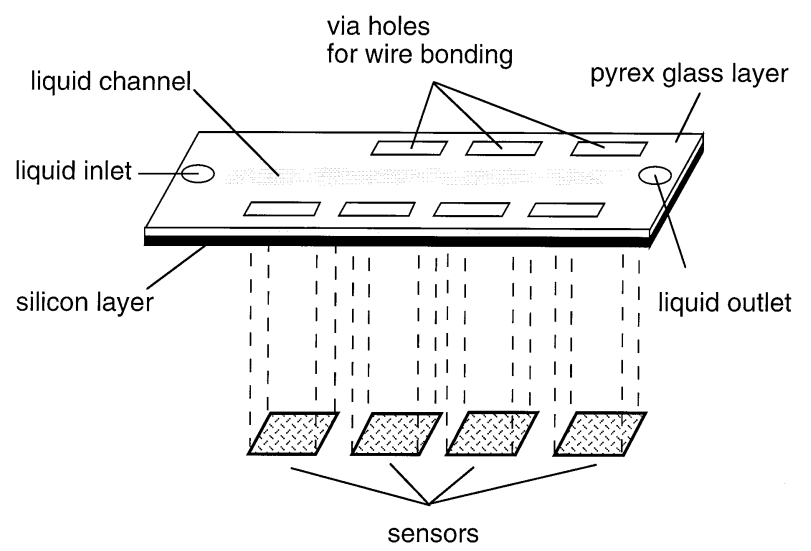

Fig. 4. Sensor carrier layout. 


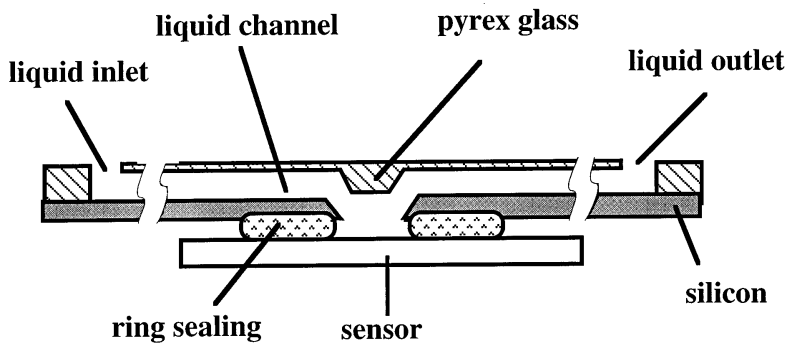

Fig. 5. Cross section along the liquid channel.

sealing. Grooves, in which epoxy can be dispensed, have been micromachined in the silicon structure. A biocompatible torr seal epoxy curable at room temperature is used. It is deposited in the silicon grooves with a programmable dispenser. The sensors are aligned and placed by means of a flipchip bonder. Electrical contacts to the sensor bond pads are realized at room temperature by ultrasonic A1 wire bonding. The bond pads are accessed by holes in the sensor carrier. Afterwards a UV curable transparent glob top epoxy DYMAX 425 is used to protect the wire bond. The transparency of the glass channel allows the investigation of the liquid flow through the channel with a microscope. All parts (e.g. silicon, pyrex glass, sensors, epoxy) in contact with the fluid are biocompatible.

\section{Sensor chips}

The following sensors have been mounted on a demonstrator sensor carrier:

- Capacitive pressure sensor: this device is a high sensitivity pressure sensor $(35 \pm 10) \mathrm{fF} \mathrm{kPa}^{-1}$ [1]. The rest capacitance is about $(4 \pm 0.5) \mathrm{pF}$.

- Temperature sensor: the temperature is sensed by means of a conventional Si diode, which is fabricated in standard $1.25 \mu \mathrm{m}$ CMOS technology [2].

- pH-ISFET sensor: the ISFET sensor is based on a $\mathrm{Ta}_{2} \mathrm{O}_{5}$ gate process for maximum sensitivity and

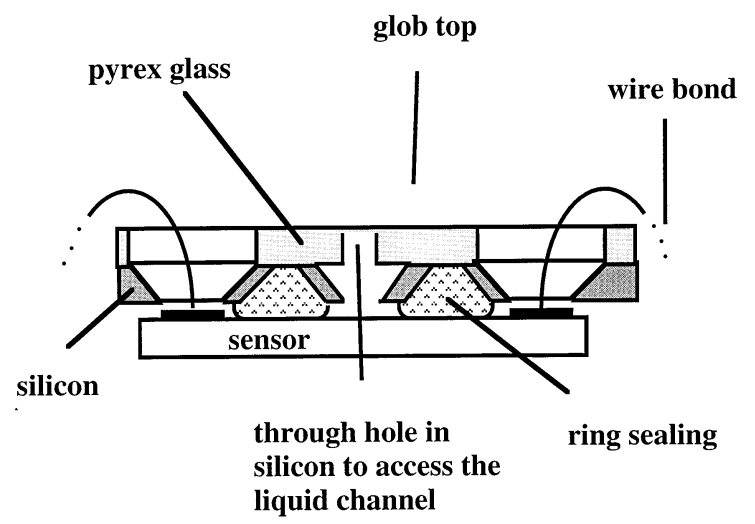

Fig. 6. Cross section perpendicular to the liquid channel.

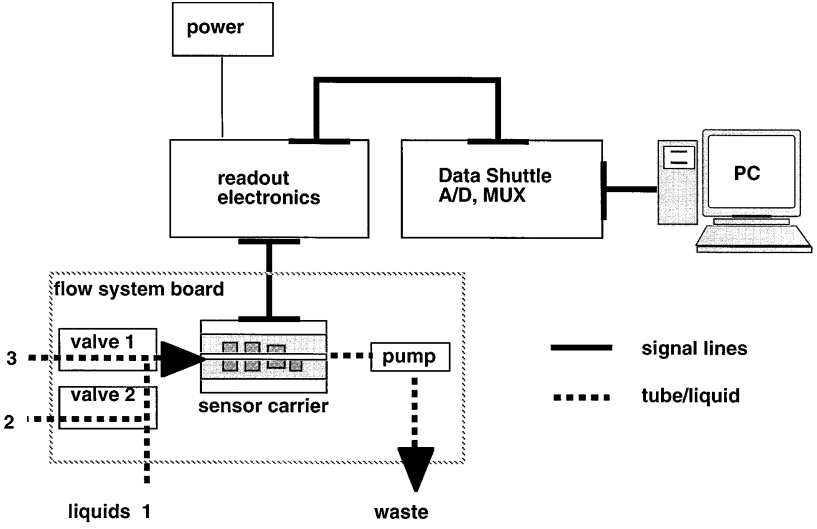

Fig. 7. Microsensor array configuration.

minimum drift [3]. The device contains two ISFETs. On one of the two ISFETs, polymer is deposited and patterned to create a longer diffusion time. During this time, the $\mathrm{pH}$ is measured differentially between the fast and slow response ISFET.

- $\mathrm{pO}_{2} / \mathrm{pCO}_{2}$ sensor: the detection principles of this combined sensor [4] are respectively a Clarktype $\mathrm{pO}_{2}$ sensor $\left(\mathrm{O}_{2}+2 \mathrm{H}_{2} \mathrm{O}+4 \mathrm{e}^{-} \leftrightarrow 4 \mathrm{OH}^{-}\right)$and a Severinghaus-type $\mathrm{pCO}_{2}$ sensor $\left(\mathrm{CO}_{2}+\mathrm{H}_{2} \mathrm{O} \leftrightarrow\right.$ $\mathrm{H}_{2} \mathrm{CO}_{3} \leftrightarrow \mathrm{H}^{+}+\mathrm{HCO}_{3}^{-}$.

\section{Interface electronics and data acquisition}

A separate printed circuit board containing the readout electronics is connected to the flow system board either directly by SIL connectors or by flatcable. This printed circuit board takes care of signal conditioning, amplification, power supply for the sensors, pump and valves and the interface to the data acquisition.

After the signal conditioning, a data shuttle performs multiplexing and A/D conversion (12-bit). A PC acquires the data and controls the complete microsensor array: manual/automatic switching of valves, data acquisition (calibration parameters, units), presentation of sensor data, etc. An overview of the system layout is given in Fig. 7.

\section{Experimental results}

Functional tests on the microsensor array have been performed in dry environment as well as with liquid samples (e.g. leakage, priming, interference, sensitivity, ...). The ability to sterilize the sensor carrier by means of $\mathrm{H}_{2} \mathrm{O}_{2}$ has been demonstrated. Two days after sterilization, no contamination was found in the nutrient. Compatibility with different salt and protein concentrations has been shown. The usability of the microsensor array for fermentation has been proven in 
a glucose mineral salt medium with $E$. coli cells. The influence of the cell growth on the sensor properties has been tested.

\section{Conclusions}

The manufacturing of a hybrid microsensor array which satifies requirements for multi-analyte biochemical analysis, is presented. The technology can be used to mount different types of sensors (e.g. potentiometric, amperometric, ...). The developed analysis system is characterized by its biocompatibility, multi-purpose and multi-user capability. In particular, low temperature processes are used for the sensor integration. The possibility of sterilization of the sensor array has been verified.

\section{Acknowledgements}

This work was performed as part of an ESA funded project. We wish to thank Mike Garvin from ESA/ESTEC (NL) and to acknowledge the valuable contribution of MSTB (UK).

\section{References}

[1] E. Peeters, Process development for 3D silicon microstructures with application to mechanical sensor devices, Thesis, Katholieke Universiteit Leuven, Belgium, 1994.

[2] F. Christiaens, E. Beyne, W. Temmerman, K. Allaert, W. Nelemans, Experimental thermal characterisation of electronic packages in a fluid bath environment, Proceedings of Eurotherm Seminar No. 45 Thermal Management of Electronic Systems, Leuven, Belgium, 1995.

[3] A. van den Berg, K. Koudelka, B.H. van der Schoot, A. Grisel, A universal on-wafer fabrication technique for diffusion limiting membranes for use in microelectrochemical amperometric sensors, Sensors and Actuators B vol. B5, Nos. 1-4, 1991.

[4] $\mathrm{Ph}$. Arquint, Integrated blood gas sensor for $\mathrm{pO}_{2}, \mathrm{pCO}_{2}$ and $\mathrm{pH}$ based on silicon technology, Thesis, University of Neuchâtel, IMT, Switzerland, 1994.

\section{Biographies}

Filip Van Steenkiste, Filip Van Steenkiste received an M.Sc. degree in electrical engineering in 1992 at the polytechnic school BME in Ghent. He received a second M.Sc. degree in applied physics in 1995 at the University of Brussels. His graduate works were respectively on an ASIC and on nonlinear characterization of optical waveguides. Since 1995, he has joined the microsystem technologies group at the Interuniversity Micro-Electronics Center (IMEC) in Leuven where he is working towards a Ph.D. Currently his research deals with a monolithic bloodgas sensors.

Kris Baert, Kris Baert was born in Leuven, Belgium on October 1960. He took his Master's Degree of electrical Engineering and $\mathrm{Ph}$. D. in Micro-electronics and Materials Science form Leuven University in 1984 and 1990 respectively. The topic of his Ph.D. work was low temperature plasma-enhanced CVD of Si materials and their applications in solar cells and TFTs. In 1988, he was a visiting scientist at the Konagai-Takahashi laboratory of the Tokyo Institute of Technology, Japan. From 1990 until 1992, he was with the Materials and Electronic Devices Laboratory of Mitsubishi Electric, Amagasaki, Japan where he worked on poly-Si TFTs for active-matrix liquid crystal displays. At present, he is with the Interuniversity Micro-Electronics Center (IMEC) in Leuven, Belgium where he is responsible for the activities on microsystem technologies. 